\title{
Preparation of Cationic Gold Nanoparticles for Gene Delivery
}

\author{
Guoping Chen ${ }^{1,2, *}$, Michiaki Takezawa ${ }^{1,2}$, Naoki Kawazoe ${ }^{1}$ and Tetsuya Tateishi ${ }^{1}$
}

${ }^{1}$ Biomaterials Center, National Institute for Materials Science, 1-1 Namiki, Tsukuba, Ibaraki 305-0044, Japan and

${ }^{2}$ Graduate School of Pure and Applied Science, University of Tsukuba, 1-1-1 Tennodai, Tsukuba, Ibaraki 305-8571, Japan

\begin{abstract}
Cationic gold nanoparticles modified with 2-aminoethanethiol, 8-amino-1-octanethiol, and 11-amino-1undecanethiol were prepared by $\mathrm{NaBH}_{4}$ reduction of $\mathrm{HAuCl}_{4}$ in the presence of thiols in water or a water/ethanol mixture solvent. The formation of gold nanoparticles depended on the kind of solvent, and the concentration of $\mathrm{NaBH}_{4}, \mathrm{HAuCl}_{4}$, and thiols. A low concentration of $\mathrm{HAuCl}_{4}$ and a high concentration of $\mathrm{NaBH}_{4}$ facilitated the formation of gold nanoparticles. A high concentration of $\mathrm{HAuCl}_{4}$ solution resulted in aggregation and a low concentration of $\mathrm{NaBH}_{4}$ did not induce the formation of gold nanoparticles. The size of the gold nanoparticles increased with an increase of the concentration of the $\mathrm{HAuCl}_{4}$ solution, while it decreased with an increase of the concentration of the $\mathrm{NaBH}_{4}$ solution. When thiols were present, the $\mathrm{HAuCl}_{4} /$ thiol ratio must be in the range of 1:1 to 1:2 for the formation of gold nanoparticles in both the water and water/ethanol mixture solvents. Modification with thiol molecules changed surface charge of the gold nanoparticles from negative to positive, and stabilized the gold nanoparticles. The cationic gold nanoparticles promoted gene transfection. A high ratio of gold nanoparticles to DNA increased transfection efficiency. However, the length of the alkyl groups had no evident effect.
\end{abstract}

\section{INTRODUCTION}

Gene transfection has been developed as a powerful technique for elucidating gene function, and for treating and controlling diseases [1]. Viral and nonviral methods can be used for gene transfection. Viral methods have high transfection efficiency. However, the viral methods have the problems of immunogenicity and induction of oncogenic responses. The nonviral methods include physical and chemical methods $[2$, 3]. Physical methods such as electroporation, particle bombardment, and fine-needle naked DNA injection have been developed to facilitate gene transfection. Chemical methods by using cationic carriers such as cationic lipids, liposomes, polyethylenimine, polyamidoamine, polypropylenimine, polyL-lysine, polyallylamine, and cationic dextran can form complexes with DNA and exhibit transfection activity. The nonviral methods have the advantages of safety, ease of preparation, reproducibility, and stability. However, the main drawbacks of nonviral-mediated gene transfection are related to low transfection efficiency. Development of new nonviral methods to facilitate high transfection efficiency is strongly desirable.

In recent years, gold and inorganic nanoparticles have been used in alternative approaches to gene transfection [412]. Cationic gold and silica nanoparticles have been used for efficient transfection of 293 T and COS-1 cells. Prow et al. used gold nanoparticles, semiconductor nanocrystals, and magnetic nanoparticles that were modified with biotinlabeled transcriptionally active PCR products for gene transfection [7]. Sandhu et al. reported gene transfection using gold nanoparticles modified with $N, N, N$-trimethyl(11-merca-

*Address correspondence to this author at the Biomaterials Center, National Institute for Materials Science, 1-1 Namiki, Tsukuba, Ibaraki 305-0044, Japan; E-mail: Guoping.CHEN@nims.go.jp ptoundecyl) ammonium chloride and alkylthiol with several chain lengths [10]. Niidome et al. reported preparation of gold nanoparticles coated with 2-aminoethanethiol and their application in gene transfection [12]. However, the effect of the alkyl group has not been elucidated. In this study, cationic gold nanoparticles modified with various thiols were prepared and used for gene transfection. The effects of length of the alkyl group in thiols, and the Au/DNA ratio were investigated.

\section{EXPERIMENTAL}

\section{Preparation of Gold Nano-Particles in Aqueous Solution}

Each of five aqueous solutions of $\mathrm{NaBH}_{4}(50 \mu \mathrm{L})$ was dropwisely added to five aqueous solutions of $\mathrm{HAuCl}_{4}(5$ $\mathrm{mL}$ ) in tubes with shaking in the dark. The 25 reaction solutions were kept shaking for another three hours at room temperature in the dark. The concentration of the $\mathrm{HAuCl}_{4}$ aqueous solution was $0.24,1.20,2.40,12.20$, and $24.00 \mathrm{mM}$ while the concentration of the $\mathrm{NaBH}_{4}$ aqueous solution was $2.64,5.28,26.4,264$, and $2640 \mu \mathrm{M}$.

\section{Preparation of 2-Aminoethanethiol-Modified Gold Nanoparticles in Aqueous Solution}

The preparation scheme of gold nanoparticles in the presence of thiols is shown in Fig. (1). 2-Aminoethanethiol was first added to an aqueous solution of $\mathrm{HAuCl}_{4}$ in a tube. Then, $50 \mu \mathrm{L}$ of $\mathrm{NaBH}_{4}$ aqueous solution was dropwisely added with shaking in the dark. The mixture solution was kept shaking for another three hours at room temperature in the dark. The concentrations of the aqueous solutions of $\mathrm{HAuCl}_{4}$ and 2-aminoethanethiol were $0.24 \sim 2.4 \mathrm{mM}$ and $0.18 \sim 7.2$ $\mathrm{mM}$, respectively. The concentration of the $\mathrm{NaBH}_{4}$ aqueous solution was $2.64,5.28$, and $26.4 \mu \mathrm{M}$. 


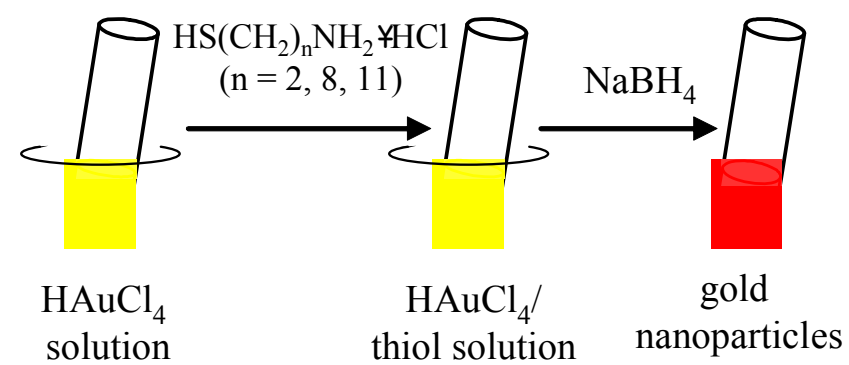

Fig. (1). Preparation scheme of thiol-modified gold nanoparticles.

\section{Preparation of Gold Nanoparticles in Mixture Solvent}

$50 \mu \mathrm{L}$ of $\mathrm{NaBH}_{4}$ solution in water/ethanol ( $\mathrm{v} / \mathrm{v}$ ratio of 1:1) was dropwisely added to $5 \mathrm{~mL}$ of $\mathrm{HAuCl}_{4}$ solution in water/ethanol (1:1) with shaking in the dark. The reaction solution was kept shaking for another three hours at room temperature in the dark. The concentration of the $\mathrm{HAuCl}_{4}$ aqueous solution was $0.24,2.40$, and $24.0 \mathrm{mM}$. The concentration of the $\mathrm{NaBH}_{4}$ aqueous solution was 5.28, 26.4, 264, and $2640 \mu \mathrm{M}$.

\section{Preparation of 2-Aminoethanethiol-Modified Gold Nanoparticles in Mixture Solvent}

$50 \mu \mathrm{L}$ of $\mathrm{NaBH}_{4}$ solution in water/ethanol (1:1) was dropwisely added to a mixture solution of $\mathrm{HAuCl}_{4}$ and 2aminoethanethiol in water/ethanol $(1: 1,5 \mathrm{~mL})$ with shaking in the dark. The reaction solution was kept shaking for another three hours at room temperature in the dark. The concentrations of the aqueous solutions of $\mathrm{HAuCl}_{4}$ and 2aminoethanethiol were $0.24 \sim 2.4$ and $0.24 \sim 4.8 \mathrm{mM}$, respectively. The concentration of the $\mathrm{NaBH}_{4}$ aqueous solution was 5.28 and $26.4 \mu \mathrm{M}$.

\section{Preparation of 8-Amino-1-Octanethiol-Modified Gold Nano-Particles in Mixture Solvent}

$50 \mu \mathrm{L}$ of $\mathrm{NaBH}_{4}$ solution in water/ethanol (1:1) was dropwisely added to a mixture solution of $\mathrm{HAuCl}_{4}$ and 8amino-1-octanethiol in water/ethanol $(1: 1,5 \mathrm{~mL})$ with shaking in the dark. The reaction solution was kept shaking for another three hours at room temperature in the dark. The concentrations of the aqueous solutions of $\mathrm{HAuCl}_{4}$, and 8amino-1-octanethiol were 0.24 2.4 mM and 0.18 7.2 mM, respectively. The concentration of the $\mathrm{NaBH}_{4}$ aqueous solution was $5.28,26.4$, and $264 \mu \mathrm{M}$.

\section{Preparation of 11-Amino-1-Undecanethiol-Modified Gold Nano-Particles in Mixture Solvent}

The 11-amino-1-undecanethiol-modified gold nanoparticles were prepared in the same way as the 8-amino-1-octanethiol-modified gold nanoparticles. The concentrations of the aqueous solutions of $\mathrm{HAuCl}_{4}, 11$-amino-1-undecanethiol, and $\mathrm{NaBH}_{4}$ were $2.40 \mathrm{mM}, 3.65 \mathrm{mM}$, and $5.28 \mu \mathrm{M}$, respectively.

\section{Purification, Size, and Zeta Potential Measurements of Gold Nanoparticles}

After 3 hours of shaking, the reaction solutions were diluted 8-fold with MilliQ water and centrifuged at 1000$12000 \mathrm{rpm}$. The grain size distributions before and after cen- trifugation were compared to determine the optimal centrifugation speed for separating the gold nanoparticles. The centrifugation speed at which the main grain size peaks did not change was used as the optimal centrifugation speed. The prepared naked and thiol-modified gold nanoparticles were purified and collected at the optimized centrifugation speed. The size of the nanoparticles was analyzed by use of a dynamic light scattering spectrophotometer (DLS 6000, Otsuka Electronics Co., Ltd, Osaka, Japan). Zeta-potential measurements were performed on a zeta potential analyzer (Delsa 440SX, Beckman Coulter, Inc., Fullerton, CA).

\section{RESULTS}

The reaction of $\mathrm{NaBH}_{4}$ and $\mathrm{HAuCl}_{4}$ was conducted by changing the concentrations of the two aqueous solutions as shown in Table $\mathbf{1}$. The gold nanoparticles could be prepared with a solution with a low concentration of $\mathrm{HAuCl}_{4}$ but not with a solution with a high concentration of $\mathrm{HAuCl}_{4}$. At high concentrations of $\mathrm{HAuCl}_{4}$, the solution turned red immediately after the addition of the $\mathrm{NaBH}_{4}$ solution. The suspension solution was unstable and gold particles aggregated within one hour. The formation of gold nanoparticles required a solution with a high concentration of $\mathrm{NaBH}_{4}$. Gold nanoparticles could not be obtained with a solution with a low concentration of $\mathrm{NaBH}_{4}$. The mean diameter of the naked gold nanoparticles was between 11.4 and $80.1 \mathrm{~nm}$. The solution with a high concentration of $\mathrm{HAuCl}_{4}$ resulted in large-sized nanoparticles. Although the gold nanoparticles formed in solutions at 26.4 and $264 \mu \mathrm{M}$ of $\mathrm{NaBH}_{4}$ were almost the same size, the highest concentration of $2640 \mu \mathrm{M}$ resulted in formation of small-sized gold nanoparticles.

Table1. Formation and Size of Gold Nanoparticles Prepared in Water with Various Concentrations of $\mathrm{HAuCl}_{4}$ and $\mathrm{NaBH}_{4}$

\begin{tabular}{|c|c|c|c|c|c|}
\cline { 1 - 5 } $\mathbf{H a u C l}_{4}(\mathbf{m M})$ & \multirow{2}{*}{$\mathbf{0 . 2 4}$} & $\mathbf{1 . 2 0}$ & $\mathbf{2 . 4 0}$ & $\mathbf{1 2 . 2}$ & $\mathbf{2 4 . 0}$ \\
\cline { 1 - 5 } $\mathrm{NaBH}_{4}(\boldsymbol{\mu M})$ & & - & - & $\times$ & $\times$ \\
\hline \hline 2.64 & - & - & - & $\times$ & $\times$ \\
\hline 5.28 & $42.9 \pm 3.2$ & $49.4 \pm 2.1$ & $76.6 \pm 5.2$ & $\times$ & $\times$ \\
\hline 26.4 & $43.4 \pm 2.5$ & $42.2 \pm 1.1$ & $80.1 \pm 6.5$ & $\times$ & $\times$ \\
\hline 264 & $11.4 \pm 1.7$ & $39.3 \pm 0.3$ & $54.3 \pm 2.2$ & $\times$ & $\times$ \\
\hline
\end{tabular}

(-): no formation of nanoparticles; $(\times)$ : aggregation.

Reduction of $\mathrm{HAuCl}_{4}$ by $\mathrm{NaBH}_{4}$ was also conducted under the presence of 2-aminoethanethiol. The concentrations of aqueous solutions of $\mathrm{HAuCl}_{4}$ and 2-aminoethanethiol were controlled in the range of $0.24 \sim 2.4 \mathrm{mM}$ and $0.18 \sim 7.2$ $\mathrm{mM}$, respectively. The concentration of the aqueous solutions of $\mathrm{NaBH}_{4}$ was $2.64,5.28$, and $26.4 \mu \mathrm{M}$. With the presence of 2-aminoethanethiol, the concentration of the $\mathrm{NaBH}_{4}$ solution did not affect the formation of gold nanoparticles. Gold nanoparticles could be prepared with all three concentrations of $\mathrm{NaBH}_{4}$. The $\mathrm{NaBH}_{4}$ concentrations of 2.64 and $5.28 \mu \mathrm{M}$, at which the gold nanoparticles did not form in the 
absence of 2-aminoethanethiol, also facilitated the formation of gold nanoparticles when 2-aminoethanethiol was present. The effects of the concentrations of $\mathrm{HAuCl}_{4}$ and 2-aminoethanethiol solutions are summarized in Table 2. The concentration ratio of $\mathrm{HAuCl}_{4} / 2$-aminoethanethiol must be in the range from $1: 1$ to $1: 2$ to facilitate the formation of gold nanoparticles. A high percentage of $\mathrm{NaBH}_{4}$ resulted in aggregation and a low percentage did not induce the formation of nanoparticles. With the presence of 2-aminoethanethiol, the gold nanoparticles formed more readily than with the absence of 2-aminoethanethiol.

Table 2. Formation of 2-Aminoethanethiol-Modified Gold Nanoparticles Prepared with Various Concentrations of $\mathrm{HAuCl}_{4}$ and 2-Aminoethanethiol in Water. The Concentration of $\mathrm{NaBH}_{4}$ was $5.28 \mu \mathrm{M}$

\begin{tabular}{|c|c|c|c|c|c|c|c|}
\hline $\mathrm{HauCl}_{4}(\mathrm{mM})$ & \multirow{2}{*}{0.24} & \multirow{2}{*}{0.36} & \multirow{2}{*}{0.48} & \multirow{2}{*}{0.96} & \multirow{2}{*}{1.2} & \multirow{2}{*}{1.8} & \multirow{2}{*}{2.4} \\
\hline Thiol (mM) & & & & & & & \\
\hline 0.18 & $\times$ & $\times$ & $\times$ & $\times$ & $\times$ & $\times$ & $x$ \\
\hline 0.24 & ० & $\times$ & $\times$ & $\times$ & $\times$ & $\times$ & $x$ \\
\hline 0.36 & o & ० & $\times$ & $\times$ & $\times$ & $\times$ & $x$ \\
\hline 0.72 & - & 0 & 0 & $\times$ & $\times$ & $\times$ & $\times$ \\
\hline 1.2 & - & - & - & 0 & 0 & $\times$ & $x$ \\
\hline 1.8 & - & - & - & ० & ० & ० & $x$ \\
\hline 2.4 & - & - & - & - & ० & 0 & 0 \\
\hline 3.6 & - & - & - & - & - & $\circ$ & ○ \\
\hline 4.8 & - & - & - & - & - & - & 0 \\
\hline 7.2 & - & - & - & - & - & - & - \\
\hline
\end{tabular}

○: formation of nanoparticles; (-): no formation of nanoparticles; $(\times)$ : aggregation.

A mixture solution of water and methanol (1:1) was used to prepare gold nanoparticles to investigate the effect of solvent on the formation of gold nanoparticles. Naked gold nanoparticles were prepared under the same conditions as those used in water except the solvent was changed from water to the mixture solvent. Similar results were obtained with both the mixture and water solvents except for the $\mathrm{NaBH}_{4}$ solution with a concentration of $2640 \mu \mathrm{M}$ (Table 3). At this concentration, the gold nanoparticles were larger than those formed in the $\mathrm{NaBH}_{4}$ solution with a concentration of $264 \mu \mathrm{M}$, which was opposite of the results obtained in water. The 2-aminoethanethiol-modified gold nanoparticles could not be prepared using the water/ethanol mixture solvent because the particles aggregated and precipitated after reaction.

The 8-amino-1-octanethiol- and 11-amino-1-undecanethiol-modified gold nanoparticles were prepared in the water/ethanol mixture solvent because 8-amino-1-octanethiol and 11-amino-1-undecanethiol could be dissolved in the mixture solvent. The preparation condition was determined by preparing the 8-amino-1-octanethiol-modified gold nanoparticles in the mixture solvent of water and ethanol (1:1). At first, the $\mathrm{NaBH}_{4}$ concentration was fixed at $5.28 \mu \mathrm{M}$. The results, shown in Table $\mathbf{4}$, are similar to those obtained with the 2-aminoethanethiol-modified gold nanoparticles prepared in water. The concentration ratio of $\mathrm{HAuCl}_{4} / 8$-amino-1octanethiol must be in the range from $1: 1$ to $1: 2$ to induce the formation of gold nanoparticles. By fixing the concentration of $\mathrm{HAuCl}_{4}$, the effect of the concentration of $\mathrm{NaBH}_{4}$ on the formation of gold nanoparticles was also investigated. The results are shown in Table 5. Gold nanoparticles aggregated at low concentrations of 8-amino-1-octanethiol and did not form at high concentrations of 8-amino-1-octanethiol. High concentrations of $\mathrm{NaBH}_{4}$ resulted in large-size gold nanoparticles. Under the condition of the mixture solvent with the presence of 8-amino-1-octanethiol, the concentrations of $\mathrm{HAuCl}_{4}, \mathrm{NaBH}_{4}$, and 8-amino-1-octanethiol affected the formation of the gold nanoparticles. The 11-amino-1-undecanethiol-modified gold nanoparticles were prepared under the same condition as that of the 8-amino-1-octanethiolmodified gold nanoparticles.

Table 3. Formation and Size of Gold Nanoparticles Prepared with Various Concentrations of $\mathrm{HAuCl}_{4}$ and $\mathrm{NaBH}_{4}$ in Water/Ethanol Mixture Solvent

\begin{tabular}{|c|c|c|c|}
\hline \multirow{2}{*}{$\mathbf{H a u C l}_{\mathbf{4}}(\mathbf{m M})$} & $\mathbf{0 . 2 4}$ & $\mathbf{2 . 4 0}$ & $\mathbf{2 4 . 0}$ \\
\cline { 1 - 3 } $\mathrm{NaBH}_{4}(\boldsymbol{\mu M})$ & & & $\times$ \\
\hline \hline 5.28 & - & - & $\times$ \\
\hline 26.4 & $40.6 \pm 1.8$ & $51.7 \pm 3.1$ & $\times$ \\
\hline 264 & $28.1 \pm 1.7$ & $46.7 \pm 2.9$ & $\times$ \\
\hline 2640 & $31.7 \pm 1.1$ & $67.7 \pm 5.2$ & \\
\hline
\end{tabular}

$(-)$ : no formation of nanoparticles; $(\times)$ : aggregation.

Table 4. Formation of 8-Amino-1-Octanethiol-Modified Gold Nanoparticles Prepared with Various Concentrations of $\mathrm{HAuCl}_{4}$ and 8-Amino-1-Octanethiol in Water/Ethanol Mixture Solvent. The Concentration of $\mathrm{NaBH}_{4}$ was $5.28 \mu \mathrm{M}$

\begin{tabular}{|c|c|c|c|c|c|c|c|}
\hline $\mathrm{HauCl}_{4}(\mathrm{mM})$ & \multirow{2}{*}{0.24} & \multirow{2}{*}{0.36} & \multirow{2}{*}{0.48} & \multirow{2}{*}{0.96} & \multirow{2}{*}{1.2} & \multirow{2}{*}{1.8} & \multirow{2}{*}{2.4} \\
\hline Thiol (mM) & & & & & & & \\
\hline 0.18 & $x$ & $\times$ & $x$ & $x$ & $x$ & $x$ & $x$ \\
\hline 0.24 & $x$ & $\times$ & $x$ & $\times$ & $x$ & $x$ & $\times$ \\
\hline 0.36 & ○ & ० & $x$ & $\times$ & $\times$ & $x$ & $\times$ \\
\hline 0.72 & - & 0 & o & $x$ & $x$ & $x$ & $x$ \\
\hline 1.2 & - & - & - & $\circ$ & ० & $\times$ & $\times$ \\
\hline 1.8 & - & - & - & ○ & ० & 0 & $\times$ \\
\hline 2.4 & - & - & - & - & ० & ० & ० \\
\hline 3.6 & - & - & - & - & - & - & ० \\
\hline 4.8 & - & - & - & - & - & - & - \\
\hline 7.2 & - & - & - & - & - & - & - \\
\hline
\end{tabular}

$\circ$ : formation of nanoparticles; (-): no formation of nanoparticles; $(\times)$ : aggregation. 
Table 5. Formation and Size of 8-Amino-1-OctanethiolModified Gold Nanoparticles Prepared with Various Concentrations of 8-Amino-1-Octanethiol and $\mathrm{NaBH}_{4}$ in Water/Ethanol Mixture Solvent. The Concentration of $\mathrm{HAuCl}_{4}$ was $2.40 \mathrm{mM}$

\begin{tabular}{|c|c|c|c|c|c|}
\cline { 1 - 4 } Thiol $(\mathbf{m M})$ & \multirow{2}{*}{$\mathbf{1 . 2}$} & $\mathbf{2 . 4}$ & $\mathbf{3 . 6}$ & $\mathbf{4 . 8}$ & $\mathbf{7 . 2}$ \\
\cline { 1 - 5 } $\mathrm{NaBH}_{4}(\boldsymbol{\mu M})$ & & & & & \\
\hline \hline 5.28 & $\times$ & $44.7 \pm 6.1$ & $39.5 \pm 4.2$ & $39.4 \pm 2.6$ & - \\
\hline 26.4 & $\times$ & $34.1 \pm 7.5$ & $39.9 \pm 3.3$ & - & - \\
\hline 264 & $\times$ & $66.3 \pm 3.1$ & $112.8 \pm 16.9$ & - & - \\
\hline
\end{tabular}

(-): no formation of nanoparticles; $(\times)$ : aggregation.

Finally, the gold nanoparticles used for gene transfection were prepared under the conditions shown in Table $\mathbf{6}$. Naked, 8-amino-1-octanethiol-modified, and 11-amino-1-undecanethiol-modified gold nanoparticles were prepared in a water/ ethanol mixture solvent, while the 2-aminoethanethiolmodified gold nanoparticles were prepared in water. The naked gold nanoparticles were negatively charged, while the modified gold nanoparticles were positively charged. The cationic thiols bound to the gold nanoparticles and changed their electrostatic properties.

The 2-aminoethanethiol-, 8-amino-1-octanethiol-, and 11-amino-1-undecanethiol-modified gold nanoparticles were used for transfection of the plasmid DNA coding EGFP. The $\mathrm{Au} / \mathrm{DNA}(\mathrm{w} / \mathrm{w})$ ratios used for transfection were 1:1, 5:1, 10:1, 15:1, and 20:1. Observation with fluorescence microscopy demonstrated that all the thiol-modified gold nanoparticles had some effect on gene transfection (Fig. 2) and the effect increased with an increase in the Au/DNA (w/w) ratio. No evident effect of the length of the alkyl groups was detected.

\section{DISCUSSION}

Gold nanoparticles were prepared by $\mathrm{NaBH}_{4}$ reduction of $\mathrm{HAuCl}_{4}$ in water or water/ethanol mixture solvents under the absence or presence of thiols. All the conditions affected the formation and size of the gold nanoparticles. A low concentration of $\mathrm{HAuCl}_{4}$ and a high concentration of $\mathrm{NaBH}_{4}$ promoted the formation of gold nanoparticles. A solution with a high concentration of $\mathrm{HAuCl}_{4}$ resulted in aggregation and a low concentration of $\mathrm{NaBH}_{4}$ did not facilitate the formation of gold nanoparticles. The size of the gold nanoparticles increased with an increase in the concentration of $\mathrm{HAuCl}_{4}$, while it decreased with an increase in the concentration of $\mathrm{NaBH}_{4}$. It is thought that the high concentration of $\mathrm{NaBH}_{4}$ might result in quick reduction of $\mathrm{HAuCl}_{4}$, which results in the formation of small-sized gold nanoparticles. The $\mathrm{NaBH}_{4}$ reduction of water might compete with the reduction of $\mathrm{HAuCl}_{4}$. The low concentration of $\mathrm{NaBH}_{4}$ solution might react with water other than $\mathrm{HAuCl}_{4}$, which could explain the failure to form gold nanoparticles in a solution with a low concentration of $\mathrm{NaBH}_{4}$.
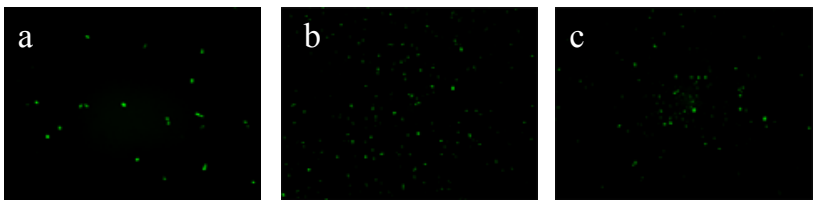

Fig. (2). Fluorescence micrographs of COS-7 cells transfected with EGFP plasmid by 2-aminoethanethiol- (a), 8-amino-1-octanethiol(b) and 11-amino-1-undecanethiol-modified (c) gold nanoparticles at $\mathrm{Au} / \mathrm{DNA}(\mathrm{w} / \mathrm{w}): 20: 1$.

With the absence of thiols, the water and water/ethanol mixture solvents showed similar effects except for the solution with the high concentration of $\mathrm{NaBH}_{4}$. At a high concentration of $\mathrm{NaBH}_{4}$, the two solvents had opposite effects.

When thiols were present, the concentration ratio of $\mathrm{HAuCl}_{4} /$ thiol was more important in the formation of gold nanoparticles than the concentrations and the kind of solvent. The $\mathrm{HAuCl}_{4} /$ thiol ratio must be in the range from $1: 1$ to $1: 2$ for forming gold nanoparticles in both water and water/ethanol mixture solvents. A high ratio of $\mathrm{NaBH}_{4}$ resulted in aggregation and a low ratio inhibited the formation of nanoparticles. The gold nanoparticles could form more readily in the presence of thiol than in the absence of thiol. This might be due to the protective effect of the aggregation of the gold nanoparticles from the thiol molecules. However, no 2-aminoethanethiol-modified gold nanoparticles formed in the water/ethanol mixture solvent because the particles aggregated and precipitated after reaction. The reason for this is not clear. The thiol molecules assembled on the surfaces of the gold nanoparticles changed the surface properties of the gold nanoparticles from a negative charge to a positive

Table 6. Preparation of Naked and Thiol-Modified Gold Nanoparticles and their Properties

\begin{tabular}{|c|c|c|c|c|c|c|c|}
\hline Measurement & \multicolumn{4}{|c|}{ Preparation Condition } & \multirow{3}{*}{ Size $(\mu \mathbf{m})$} & \multirow{2}{*}{\multicolumn{2}{|c|}{ Zeta Potential (mV) }} \\
\hline Date & \multirow{2}{*}{ Solvent } & \multicolumn{3}{|c|}{ Concentration } & & & \\
\hline Thiol & & Thiol (mM) & $\mathrm{HAuCl}_{4}(\mathrm{mM})$ & NaBH4 $(\boldsymbol{\mu M})$ & & pH 4.0 & pH 7.0 \\
\hline naked & water/ethanol & 0 & 0.24 & 26.4 & $40.6 \pm 1.8$ & $-10.0 \pm 1.2$ & $-9.6 \pm 3.1$ \\
\hline 2-aminoethanethiol & water & 3.65 & 2.40 & 5.30 & $31.6 \pm 5.6$ & $14.3 \pm 4.7$ & $0.1 \pm 0.2$ \\
\hline 8-amino-1-octanethiol & Water/ethanol & 3.65 & 2.40 & 5.30 & $35.9 \pm 1.8$ & $6.6 \pm 4.1$ & $0.1 \pm 0.1$ \\
\hline 11-amino-1-undecanethiol & Water/ethanol & 3.65 & 2.40 & 5.30 & $34.3 \pm 3.8$ & $5.7 \pm 2.8$ & $0.3 \pm 0.6$ \\
\hline
\end{tabular}


charge. The assembled thiol molecules could also protect the aggregation of the gold nanoparticles and stabilize the system.

The cationic gold nanoparticles could compact the DNA plasmid and facilitated transfection of the EGFP plasmid. A high ratio of gold nanoparticles to DNA increased transfection efficiency but the length of the alkyl groups had no evident effects. It has been reported that the size of the nanoparticles and their ratios to DNA have been shown to affect transfection efficiency. Niidome et al. reported that a high $\mathrm{Au} / \mathrm{DNA}$ ratio could increase transfection efficiency [12]. Prow et al. reported that the larger the nanoparticles, the better the transfection efficiency [7]. Sandhu et al. reported that maximum transfection was achieved at an Au/plasmid mole ratio of 2200:1 [10].

\section{CONCLUSIONS}

Gold nanoparticles modified with 2-aminoethanethiol, 8amino-1-octanethiol, and 11-amino-1-undecanethiol were prepared by $\mathrm{NaBH}_{4}$ reduction of $\mathrm{HAuCl}_{4}$ in water or water/ethanol mixture solvents under the presence of respective thiols. Modification of the thiol molecules changed the electrostatic properties of the gold nanoparticles from a negative charge to a positive charge. The cationic gold nanoparticles interacted with negatively charged plasmid DNA and their complex could be used for gene transfection. A high ratio of gold nanoparticles to DNA increased transfection efficiency.

\section{ACKNOWLEDGEMENT}

This work was supported by the Ministry of Education, Culture, Sports, Science and Technology of Japan.

\section{REFERENCES}

[1] Luo D, Saltzman WM. Synthetic DNA delivery systems. Nat Biotechnol 2000; 18(1): 33-7.

[2] Romano G. Current development of nonviral-mediated gene transfer. Biochem Biophys Res Commun 2007; 362(4): 835-841.

[3] Niidome T, Huang L. Gene therapy progress and prospects: nonviral vectors. Gene Ther 200; 9(24): 1647-52.

[4] Cai X, Conley S, Naash M. Nanoparticle applications in ocular gene therapy. Drug News Perspect 2007; 20(4): 227-31.

[5] Noh SM, Kim WK, Kim SJ, et al. Enhanced cellular delivery and transfection efficiency of plasmid DNA using positively charged biocompatible colloidal gold nanoparticles. Biochim Biophys Acta 2007; 1770(5): 747-52.

[6] Swami A, Kurupati RK, Pathak A, et al. A unique and highly efficient non-viral DNA/siRNA delivery system based on PEIbisepoxide nanoparticles. Biochem Biophys Res Commun 2007; 362(4): 835-41.

[7] Prow T, Smith JN, Grebe R, et al. Construction, gene delivery, and expression of DNA tethered nanoparticles. Mol Vis 2006; 12: 60615 .

[8] Rosi NL, Giljohann DA, Thaxton CS, et al. Oligonucleotidemodified gold nanoparticles for intracellular gene regulation. Science 2006; 312(5776): 1027-30.

[9] Thomas M, Klibanov AM. Conjugation to gold nanoparticles enhances polyethylenimine's transfer of plasmid DNA into mammalian cells. Proc Natl Acad Sci USA 2003; 100(16): 9138-43.

[10] Sandhu KK, McIntosh CM, Simard JM, et al. Gold nanoparticlemediated transfection of mammalian cells. Bioconjug Chem 2002; 13(1): 3-6

[11] Kneuer C, Sameti M, Bakowsky U, et al. A nonviral DNA delivery system based on surface modified silica-nanoparticles can efficiently transfect cells in vitro. Bioconjug Chem 2000; 11(6): 92632.

[12] Niidome T, Nakashima K, Takahashi H, Niidome Y. Preparation of primary amine-modified gold nanoparticles and their transfection ability into cultivated cells. Chem Commun 2004; (17): 1978-9. 